\title{
Untersuchungen über die Wassermannsche Reaktion bei Paralytikern.
}

\author{
Von \\ E. Nathan und R. Weichbrodt.
}

(Aus der Dermatologischen [Geheimrat Prof. K. Herxheimer] und der Psychiatrischen [Prof. Kleist] Universitätsklinik in Frankfurt a. M.)

\section{(Eingegangen am 16. März 1921.)}

Daß bei der progressiven Paralyse die Wassermannsche Reaktion im Blut und Liquor so gut wie immer mehr oder minder stark positiv ausfällt, wird so allgemein angenommen, daß beim Fehlen der Reaktionen Zweifel an der Richtigkeit der Diagnose auftreten.

So schreibt Nonne: ,Fehlen der WaR. im Blute läßt starke Bedenken gegen die Annahme einer Paralyse aufkommen, schließt aber eine solche nicht absolut aus." Vom Liquor der Paralytiker sagt er weiter, daß er bei der Originalmethode in $85-90 \%$, bei der Auswertung sogar in 100\% positiv ausfalle. Er betont aber mit Recht, daß die Klinik uns weiter Führerin in der Diagnose bleiben müsse und werde, und daß nichts verkehrter sein würde, als bei der Differentialdiagnose eines dubiösen Falles bewährte klinische Erfahrungen zugunsten der serologischen, mikroskopischen und chemischen Untersuchungsmethoden zurückzuschieben, eine Ansicht, die sich auch, wenigstens was die WaR. betrifft, mehr und mehr in der Diagnostik der Syphilis durchsetzt. Aber wie das Fehlen der Reaktionen nicht immer eine Paralyse ausschließen läßt, so finden sich auch mitunter positive WaR. bei psychischen Störungen, die nicht paralytische sind. Krüger weist auf die Möglichkeit hin, daß die „4 Reaktionen“ als Ausdruck einer sekundären Syphilis komplizierend einen andersartigen nervösen oder psychischen Prozeß begleiten können, meint aber, daß derartige Fälle praktisch zu den größten Seltenheiten gehören. Nun hat aber Geelvink im November 1920 in der Sitzung der Vereinigung Frankfurter Neurologen und Psychiater über 4 Fälle berichtet, von denen allein 3 innerhalb eines Jahres zur Beobachtung kamen. Es handelte sich in allen Fällen um 8-10 Jahre zurückliegende syphilitische Infektionen, und alle „4 Reaktionen" waren positiv ausgefallen. Bei Fehlen aller körperlichen organisehen Symptome lagen psychogene Krankheitsbilder bzw. Reaktionen vor, die durch ein gerichtliches Verfahren ausgelöst waren. Nach Abklingen dieser Situationspsychosen war der Verdacht einer beginnenden Paralyse durch nichts mehr zu rechtfertigen. Ob derartige Spätsyphilitiker mit positivem Liquorbefund später einmal der Paralyse verfallen - der sehr angreifbare Ausdruck Präparalyse ist für derartige Fälle gebräuchlich -- ist eine Frage, zu deren Beantwortung die bisherige Beobachtung noch nicht ausreicht. Jedenfalls beweisen derartige Fälle, daß ein zufälliges Zusammentreffen der „4 Reaktionen" mit einer nicht syphilitischen Psychose keineswegs etwas abnorm Seltenes, sondern ein Vorkommnis ist, das durchaus ernstlicher Erwägung bedarf und forensisch von Bedeutung sein kann. 
E. Nathan u. R. Weichbrodt: Untersuch, über d. Wassermannsche Reakt. usw. 309

Nun ist das Dogma, daß die WaR. im Blut der Paralytiker in $100 \%$ positiv ist, durch die letzte Veröffentlichungen Kafkas ins Wanken gekommen. In 18,7\% seiner Fälle, die zu gleicher Zeit lumbal - und venäpunktiert wurden, war die WaR. im Blutserum deutlich schwächer als die der Rückenmarksflüssigkeit. Kafka wirft dabei die Frage auf, ob die Ergebnisse der WaR. im Blut und Liquor überhaupt vergleichbar wären, und er weist darauf hin, daß auch bei der Iritis specifica das Kammerwasser positiv reagieren, während das Blut negativ sein kann. F. Plaut konnte an seinem Material die Kafkasehen Erfahrungen nicht bestätigen. Unter 1420 Fällen hatten nur 16 eine negative WaR. im Blut, von diesen 16 Fällen erwiesen sich 8 als Fehldiagnosen, so daß nur 8 Fälle, also $0,6 \%$ übrig blieben. Bei keinem dieser 8 Fälle war im Liquor mit $0,2 \mathrm{ccm}$ eine positive WaR. zu erzielen, sondern erst bei Auswertung mit höheren Dosen. Pla ut meint, wenn schon einmal bei einer Paralyse die WaR. im Blut fehle, so fehle sie auch im Liquor, oder sie wäre jedenfalls so sohwach entwickelt, daB sie sich erst bei höheren Liquordosen nachweisen lasse.

Ohne auf die Kontroverse zwischen Kafka und Plaut weiter einzugehen, möchten wir uns unter Hinweis auf die von Boas, Kafka und Plaut zusammengestellte Literatur erlauben, über unsere seit über 3 Jahren durchgeführten einschlägigen Untersuchungen zu berichten, die von folgenden Fragestellungen ausgingen:

1. Ist in allen Fällen von Paralyse die WaR. im Blut und Liquor unter Berücksichtigung der Auswertung nach oben positiv? 2. Läßt sich die WaR. bei Paralytikern durch die verschiedensten Behandlungsarten beeinflussen? 3. Kann aus der Stärke der WaR, auf den Krankheitszustand und Krankheitsverlauf geschlossen werden? 4. Bestehen Beziehungen zwischen den Treponemenbefunden und der Stärke der WaR.?

Zur Beantwortung dieser Fragen stehen uns die Befunde von 310 Paralytikern zur Verfügung, von denen die WaR. im Institut für experimentelle Therapie und im Laboratorium der Dermatologischen Universitätsklinik ausgeführt wurde. Von diesen Fällen haben wir außerdem in eigenen besonderen Untersuchungen bei 150 Fällen Blut und Liquor des öfteren untersucht, und zwar, u m quantitati v vergleichbare Werte zu bekommen, diejenige Serum - bzw. Liquorverdünnung festgestellt, bei der noch eine komplett positive WaR. festzustellen war. Dabei wurden die Sera und Lumbalflüssigkeiten des gleichen Patienten immer zusammen untersucht, um vergleichbare Resultate zu haben. Bei der Auswertung der Sera gingen wir, da uns vergleichende Untersuchungen mit absteigenden Serum- und Extraktmengen in Übereinstimmung mit früheren Erfahrungen von Blumenthal und Tsakalotis sowie F. Lesser u. a. die Va. riation der Serummenge als das zweckmäßigere Verfahren gezeigt hatten, derart vor, da $\beta$ wir absteigende Serummengen, beginnend mit $0,25 \mathrm{ccm}$ einer 10 fachen Serumverdünnung, $(0,025-0,015-0,01,0,005-0,003-0,002-0,001-0,0006-$ $0,0004-0$ in Volumen $0,25 \mathrm{ccm})$ mit konstanten Extraktmengen $(0,25 \mathrm{ccm}$ eines 6 fach mit physiologischer NaCl-Lösung fraktioniert verdünnten alkoholischen cholesterinierten Rinderherzextraktes) unter Zusatz von $0,25 \mathrm{~cm} 10$ fach verdünnten frischen Meerschweinchenserums mischten, und nach einstündiger Digestion im Brutschrank bei $37 \%$ das hämolytische System $(0,25 \mathrm{ccm}$ Hammelblutkörperchen-Aufschwemmung $+0,25 \mathrm{~cm}$ der Amboceptorverdünnung) za* 
setzten. Ansetzung von Serum- und Extraktkontrollen wie üblich. Die Ablesung erfolgte nach $1-1^{1 / 2}$ stündigem Aufenthalt im Brutschrank bei $37^{\circ}$.

Die Liquoruntersuchung wurde im Prinzip gleichartig ausgeführt, nur wurden beginnend mit $0,25 \mathrm{com}$ unverdünnten Liquors folgende Verdünnungen angesetzt: $0,25-0,15-0,1-0,05-0,03-0,02-0,01-0,006-0,004-0$.

$\mathrm{Da}$ also Serum und Liquor in Vierteldosen zur Untersuchung kamen, mußte die geringste noch komplett hemmende Dose mit 4 multipliziert werden, und die so erhaltenen Werte sind unseren Berechnungen zugrunde gelegt.

In den meisten Fällen reagierte der Liquor in Mengen unter $0,1 \mathrm{ccm}$, das Seru m reagierted urchschnittlich 10-15 mal stärker. Reaktionen mit $0,00048 \mathrm{ccm}$ Serum und $0,008 \mathrm{ccm}$ Liquor, wie wir sie z. B. bei einer juvenilen Paralyse sahen, gehörten zu den Ausnahmen. Wenn ach meist das Serum viel stärker als der Liquor reagierte, so fanden sich doch auch Fälle, wo die Reaktion im Liquor stärker als im Serum ausfiel, ja sogar bei stark positiver Liquorreaktion eine negative Serumreaktion gefunden wurde. In einigen Fällen war der Liquor auch bei Auswertung nach oben völlig negativ, obwohl die Serumreaktion sehr stark ausfiel. In einem Falle z. B. reagierten noch $0,00048 \mathrm{ccm}$ Serum deutlich positiv bei negativer Liquorreaktion. Derartige Befunde sprechen ja dafür, da $B$ die WaR. im Blut nicht oder nicht allein durch die Treponemen im Hirn, sondern durch Herde an anderen Stellen des Körpers bedingt ist, was Plaut immer vermutet und was Jahnels Nachweis von Treponemen in der Aorta eines Paralytikers sehr wahrscheinlich gemacht hat. Als Beweis dafür, da $B$ die WaR. des Blutes und Liquors an verschiedenen Orten entsteht, könnten auch die therapeutischen Erfahrungen bei Paralytikernverwertet werden, denn die WaR. im Blut wird häufigmit einer mittelstarken Salvarsankur zum Schwinden gebracht, während sie im Liquor, wenn überhaupt, meist nur mit sehr großen Salvarsandosen beseitigt werden kann.

Was nun die Beantwortung der ersten Frage nach der Konstanz des Vorkommens einer positiven WaR. im Blut und Liquor bei Paralytikern betrifft, sollen zunächst alle jene Fälle ausgeschlossen werden, die während der Beobachtung oder kurz vorher irgendwie behandelt worden waren. Wo bei den nun folgenden Zusammenstellungen nichts erwähnt ist, liegt kein Sektionsergebnis vor.

Fall 1. Hermann Sch. 47 Jahre.

19. VI. 1917. WaR, im Blut neg., in Liquor pos. 0,2.

Fall 2. Heinrich B. 46 Jahre.

4. X. 1918. WaR. im Blut neg, im Liquor pos. 0,2.

Fall 3. Friedr. R. 54 Jahre.

9. XII. 1915. WaR. im Blut neg., im Liquor pos. 0,2.

21. XII. 1915. Derselbe Befund.

22. XII. 1915. Exitus, Sektion bestätigte die Diagnose. 
Untersuchungen über die Wassermannsche Reaktion bei Paralytikern. 311

Fall 4. Julius v. R. 38 Jahre.

1. VI. 1917. WaR. im Blut neg., im Liquor pos. 0,2.

4. XII. 1917. Exitus, Sektion bestätigte die Diagnose.

Fall 5. Jakob J., 49 Jahre.

13. V. 1918. WaR. im Blut neg., im Liquor pos. 0,4.

19. V. 1918. Exitus, die Sektion bestätigte die Diagnose.

Fall 6. August Sch. 49 Jahre.

21. VI. 1918. WaR. im Blut neg., im Liquor pos. 0,4.

6. VII. 1918 derselbe Befund.

12. VIII. 1918. Exitus, die Sektion bestätigte die Diagnose.

Fall 7. Peter K. 59 Jahre.

1. XI. 1916. WaR. im Blut neg., im Liquor pos. 1,0 .

Fall 8. Georg G. 39 Jahre.

21. I. 1918. WaR. im Blut pos., im Liquor pos. 0,2 .

6. II. 1918. Derselbe Befund.

1. III. 1918. WaR. im Blut neg., im Liquor pos. 0,016.

2. III. 1918. Exitus, die Sektion bestätigte die Diagnose.

Fall 9. Valentin M. 53 Jahre.

21. IX. 1916. WaR. im Blut pos., im Liquor pos. 0,2.

14. XI. 1918. WaR. im Blut sehr schwach pos., im Liquor pos. 0,2.

2. XII. 1918. Exitus, die Sektion bestätigte die Diagnose.

Fall 10. Gustay D. 55 Jahre.

9. XI. 1918. WaR. im Blut pos., im Liquor pos. 0,2.

7. I. 1919. Derselbe Befund.

20. VI. 1919. WaR. im Blut neg., im Liquor pos. 0,2.

11. VIII. 1919. Exitus, die Sektion bestätigte die Diagnose.

Fall 11. August S. 38 Jahre.

24. V. 1917. WaR. im Blut pos., im Liquor pos. 0,2.

9. III. 1918. WaR. im Blut neg, im Liquor pos. 0,016.

13. III. 1918. Exitus, die Sektion bestätigte die Diagnose.

Fall 12. Vincenz H. 53 Jahre:

23. VII. 1914. WaR. im Blut pos,, im Liquor pos. 0,2.

30. IV. 1917. WaR. im Blut neg., im Liquor neg.

10. V. 1917. WaR. im Blut schwach pos., im Liquor pos. 0,2 .

7. I. 1918. Exitus, die Sektion bestätigte die Diagnose.

Fall 13. Anton M. 41 Jahre.

3. V 1917. WaR. im Blut schwach pos, im Liquor neg.

10. V. 1917. WaR. im Blut pos., in Liquor pos. 0,2.

22. V. 1917. Exitus, die Sektion bestätigte die Diagnose, es fanden sich zahlreiche bewegliche Treponemen.

Fall 14. Katharina A. 47 Jahre.

20. VII. 1918. WaR. im Blut pos., im Liquor pos. 0,2.

3. IX. 1918. Derselbe Befund.

9. XI. 1918. WaR. im Blut sehr schwach pos., im Liquor pos. 0,2.

11. XI. 1918. Exitus, die Sektion bestätigte die Diagnose, es fanden sich zahlreiche gut bewegliche Treponemen. 
Fall 15. Georg Gr. 56 Jahre.

21. X. 1919. WaR. im Blut schwach pos., im Liquor pos. 0,4.

2. IV. 1920. WaR. im Blut neg., im Liquor pos. 0,4.

3. IV. 1920. Tod im paralytischen Anfall, bei der Gehirnsektion fanden sich zahlreiche bewegliche Treponemen.

Fall 16. Moritz K. 58 Jahre.

5. IV. 1917. WaR. im Blut pos., im Liquor pos. 0,2.

12. VII. 1917. WaR. im Blut neg., im Liquor pos. 0,2.

Fall 17. Heinrich B. 42 Jahre.

4. XII. 1916. WaP.. im Blut neg., imLiquor bei 1,0 Andeutung von pos. Reaktion.

6. XII. 1916. Exitus, die Sektion bestätigte die Diagnose.

Wir haben von den unbehandelten Fällen in dieser Zusammenstellung nur die angeführt, die zu irgendeiner Zeit bei negativer Blutreaktion eine positive Liquorreaktion hatten. Außerdem wären noch 5 Fälle zu erwähnen, bei de nen die Blu treak tion positiv und die Liquorreaktionen auch bei Auswertung nach oben negativ a usfielen. In einem dieser Fälle handelt es sich um eine stationäre Paralyse. Fälle, bei denen Blut-und Liquorreaktion ständig negativ waren, haben wir unter unseren unbehandelten Fällen nicht aufzuweisen. Jedenfalls geht aus dieser Aufstellung hervor, dab auch bei unbehandelten Fällen die WaR. nicht so gut wie immer im Blut und Liquor positiv a usfällt, daß vielmehr die eine oder andere Reaktion gelegentlich fehlen kann. Welche aber von beiden Reaktionen, ob die Blutreaktion oder die Liquorreaktion häufiger fehlt, läßt sich ohne weiteres nicht entscheiden, denn dazu sind einige Fälle nicht häufig genug untersucht worden. Wie wir ferner an einigen Fällen sahen, schwanken die Reaktionen bei manchen Kranken im Verlaufe der Krankheit sehr, und es kann sehr wohl sein, daß bei allen Kranken zu irgendeiner Zeit beide Reaktionen positiv waren, wie ja a uch bei jenen Fällen, deren Blut und Liquor bei einmaliger Untersuchung positiv reagierte, eine negative Schwankung uns entgangen sein kann. Vielleicht lassen sich aus diesen spontanen Schwankungen auch die Divergenzen zwischen Kaf ka und Pla ut teilweise erklären.

Diese Zufälligkeiten, wie sie uns hier bei der Paralyse entgegentreten, sind auch sehr zu berücksichtigen beim Studium des Liquors der frisehen Syphilis. So konnten wir bei einem Fall, der im März 1919 eine Luesinfektion akquirierte, von Anfang Mai bis Ende Dezember 1919 sehr häufig Blut und Liquor untersuchen. Während nun mit dem Ausbruch der Sekundärsyphilis die WaR. im Blutserum bei häufiger Untersuchung positiv wurde und blieb, zeigte der Liquor bei gleichzeitiger Untersuchung immer negative WaR. Nur einmal, a m 3. 7. 19, wardas Blut bei 0,04 und der Liquor bei 0,024 po- 
siti v. 14Tage darauf war derLiquor wieder völlig negativ, und wir fanden nie mehr eine positive Liquorreaktion, während das Blut positiv blieb.

Wenn es sich auch nur um eine Beobachtung handelt, so können wir doch aus ihr erkennen, daß wir auch vorübergehende Veränderungen des Liquors im Sekundärstadium der Syphilis häufiger annehmen müssen, als gefunden werden, und daß auch über diese Frage nur durch oft wiederholte Untersuchungen Klarheit zu bekommen ist, was allerdings nur in wenigen Fällen zu erreichen sein wird.

Wenn wir nun die Fälle betrachten, die kurz vor der Aufnahme oder während des Aufenthaltes in der Anstalt behandelt worden waren, so zeigte es sich, daß jede Behandlungsart für längere oder kürzere Zeit eine der beiden oder mitunter auch beide Reaktionen zum Schwinden bringen kann, und zwar konnten wir die Beobachtung machen, da $\beta$ in den meisten Fällen die Blutreaktion viel leichter als die Liquorreaktion zu beeinflussen ist. Es gab wohl Fälle, wo wir mit keiner Therapie die Reaktionen beeinflussen konnten, aber bei den meisten Fällen sahen wir die Reaktionen für kürzere oder längere Zeit schwinden oder schwächer werden. Bei den Salvarsanpräparaten waren meist sehr hohe Dosen nötig, um den Liquor zu beeinflussen, was aber auch bei hohen Dosen nicht in allen Fällen gelang. Bei der Fiebertherapie sahen wir nur gute Einwirkungen, wenn die Temperaturen sehr hoch gestiegen waren. Es würde zu weit führen, alle Fälle hier vorzubringen, wir müssen uns mit einigen Proben begnügen:

Fall 18. Siegmund M. 39 Jahre.

Vor der Aufnahme 2 Kuren mit je 4,0 g Silbersalvarsan.

Am 14. IV. 1920 aufgenommen.

20. 4. 1920. WaR. im Blut neg., im Liquor pos. 0,2.

10. V. 1920. Derselbe Befund.

30. VI. 1920. WaR. im Blut neg. Andeutung von pos., im Liquor pos. 0,2.

7. VII. 1920. Derselbe Befund.

28. X. 1920. WaR. im Blut pos., im Liquor pos. 0,2.

25. XI. 1920. Exitus, die Sektion bestätigte die Diagnose.

Fall 19. Margarete O. 40. Jahre.

Im September 1918 mit 4,8 Sulfoxylat behandelt.

26. III. 19I8. WaR. im Blut pos. 0,02, im Liquor pos. 0,2 .

25. VI. 1918. WaR, im Blut pos. 0,12, im Liquor pos. 0,12.

5. IX. 1918. WaR. im Blut pos. 0,04, im Liquor pos. 0,5.

15. IX. 1918. WaR. im Blut pos. 0,06, im Liquor pos. 0,5 .

8. XI. 1918. WaR. im Blut neg., im Liquor neg.

Seitdem ist Blut und Liquor bei fast monatlicher Untersuchung bis auf den heutigen Tag negativ.

Fall 20. Johannes H. 37 Jahre.

Mit 3,0 g Silbersalvarsan behandelt.

8. V. 1918. WaR. im Blut pos. 0,02, im Liquor pos. 0,12, im Hirnliquor pos. 0,4 . 
29. VI. 1918. WaR. im Blut pos. 0,02, im Liquor pos. 0,2.

28. VII. 1918. WaR. im Blut pos. 0,2, im Liquor pos. 0,8.

1. VIII. 1918 wurde H. gebessert entlassen, am 28. XI. 1919 stellte er sich zur Untersuchung vor. WaR. im Blut neg., im Liquor pos. 0,6. Er verzog von Frankfurt, so daß weitere Untersuchungen nicht möglich waren, es soll ihm aber nach dem Bericht der Ehefrau gut gehen.

Fall 21. Jakob M. 63 Jahre,

Im Dezember 1918 und April 1920 mit Sulfoxylat behandelt.

12. IV. 1917. WaR. im Blut pos., im Liquor pos. 0,2 .

26. III. 1918. WaR. im Blut pos. 0,2, im Liquor pos. 0,2.

3. VII. 1919. WaR. im Blut neg., im Liquor pos. 0,4.

24. X. 1919. WaR. im Blut neg., im Liquor pos. 0,08.

24. II. 1920. WaR. im Blut neg., im Liquor pos. 0,2.

12. III. 1920. WaR. im Blut pos. 0,2, im Liquor pos. 0,2.

24. III. 1920. WaR. im Blut pos. 0,06, im Liquor pos. 0,12.

16. IV. 1920. WaR. im Blut pos, 0,06, im Liquor 0,12.

17. VI. 1920. WaR. im Blut neg., im Liquor pos. 0,2.

3. VII. 1920. WaR. im Blut neg., im Liquor pos. 0,4.

25. X. 1920. WaR. im Blut pos., 0,02, im Liquor pos. 0,2.

M. lebt außerhalb der Anstalt in leidlicher Remission.

Fall 22. Julius Sch. 47 Jahre.

Im November mit Sulfoxylat intramuskulär behandelt.

14. IX. 1918. WaR. im Blut pos. 0,004, im Liquor pos. 0,04 .

28. XI. 1918. WaR. im Blut neg., im Liquor neg.

12. XII. 1918. WaR. im Blut neg., im Liquor pos. 0,016 .

30. XII. 1918. WaR. im Blut pos. 0,02, im Liquor pos. 0,012 .

2. I. 1919. Exitus, die Sektion bestätigte die Diagnose.

Fall 23. Wilhelm R. 35 Jahre.

Im Mai 1918 mit Sulfoxylat behandelt.

8. V. 1918. WaR. im Blut pos. 0,02, im Liquor pos. 0,12.

29. VI. 18. WaR. im Blut pos. 0,1, im Liquor pos. 0,2 .

6. VII. 1918. WaR. im Blut neg., im Liquor pos. 0,2.

12. XII. 1918. WaR. im Blat neg., im Liquor pos. 0,4 .

6. I. 1919. WaR. im Blut neg., im Liquor pos 1,0.

15. V. 1919. WaR. im Blut neg. im Liquor pos. 0,2.

10. X. 1919. WaR. im Blut neg., im Liquor pos. 0.2.

21. XII. 1919. WaR. im Blut neg., im Liquor pos. 0,12.

8. I. 1920. Exitus, die Sektion bestätigte die Diagnose.

Fall 24. Simon G. 34. Jahre.

Mit Malaria infiziert.

30. XII. 1918. WaR. im Blut pos. 0,04. im Liquor pos. 0,12.

31. I. 1919. WaR. im Blut pos. 0,2, im Liquor pos. 0,6.

24. II. 1919. WaR. im Blut neg., im Liquor neg.

Bei sehr häufigen Untersuchungen zeigten Blut und Liquor bis zum Tode negative Reaktionen.

25. X. 1919. Exitus infolge Sepsis, die Sektion bestätigte die Diagnose Paralyse.

Fall 25. Heinrich A. 31 Jahre.

Mit Malaria infiziert.

29. VI. 1918. WaR. im Blut pos. 0,04, im Liquor pos. 0,12.

9. VII. 1918. WaR. im Blut neg., im Liquor pos. 0,12. 
28. IX. 1918. WaR. im Blut Andeutung von pos. Reaktion, im Liquor pos. 0,12.

18. XI. 1918. WaR. im Blut neg., im Liquor pos. 0,04 .

12. XII. 1918. WaR. im Blut neg., im Liquor neg.

30. XII. 1918. WaR. im Blut neg., im Liquor pos. 0,4.

30. I. 1919. WaR. im Blut neg., im Liquor pos. 0,12.

10. IV. 1919. WaR. im Blut neg., im Liquor neg.

16. VI. 1919. WaR. im Blut neg., im Liquor neg.

27. VIII. 1920. WaR. im Blut neg., im Liquor neg.

Der Kranke befindet sich in einer sehr guten Remission, ist seit dem 17. IV.1919 außerhalb der Anstalt und stellt sich zur Untersuchung öfters vor. Er hat seinen Beruf als Möbeltischler aufgegeben und ist als Schreiber voll er. werbsfähig.

Fall 26. Heinrich St. 49 Jahre.

Im Dezember 1919 mit Recurrens behandelt, später mehrere Sulfoxylatkuren.

22. VII. 1918. WaR. im Blut pos., im Liquor pos. 0,2.

12. II. 1919. WaR. im Blut pos., im Liquor pos. 0,2 .

5. XI. 1919. WaR. im Blut pos. 0,08, im Liquor pos. 0,1.

12. I. 1920. WaR. im Blut pos: 0,1, im Liquor pos. 0,4 .

15. IV. 1920. WaR. im Blut pos. 0,06. im Liquor pos, 0,12.

16. VII. 1920. WaR. im Blut 0,2, im Liquor pos. 0,6 .

2. XI. 1920. WaR. im Blut pos. 0,08, im Liquor pos. 0,12.

15. I. 1921. WaR. im Blut Andeutung von pos. Reaktion, im Liquor pos. 0,8 .

St. ist seit dem 29. I. 1920 entlassen und stellt sich des öfteren zur Untersuchung vor.

An diesen wenigen Beispielen zeigt es sich deutlich, $\mathrm{da} B$ die verschiedensten Behandlungsarten die WaR. im Blut und Liquor zu beeinflussen vermögen, daß die eine oder andere Reaktion, mitunter auch beide für kürzere oder längere Zeit negativ werden können. In einigen Fällen sahen wir die Reaktionen jahrelang negativ bleiben, und nicht nur die WaR. im Blut und Liquor war negativ geworden, sondern auch die Eiweißreaktionen, und es bestand keine Lymphocytose mehr. Diese Tatsache ist aber für die Diagnostik sehr wichtig, denn wenn wir bei einem paralyseverdächtigen Kranken das Fehlen aller ,4 Reaktionen" feststellen müssen, so werden wir zuerst nachzuforschen haben, ob der Kranke nicht energisch behandelt worden ist. Von den Paralytikern, die z. B. Raecke im Jahre 1913 mit Salvarsan behandelt hat, konnten wir jetzt noch bei 4 Kranken das Fehlen aller 4 Reaktionen feststellen.

Diese Erörterung führt uns gleich zu der nächsten Frage, ob der Ausfall der WaR. Rückschlüsse auf den Krankheitszustand erlaubt. Wenn wir von den wenigen Fällen absehen, bei denen es durch energische Kuren gelungen ist, die WaR. im Blut und Liquor für Jahre zum Schwinden zu bringen, und bei denen ein Stillstand der Erkrankung klinisch eintrat, muß man gestehen, daB die WaR. nicht als zuverlässiger Maßstab für den Grad der Erkrankung angesehen werden kann. Wir haben Fälle beobachten können, wo, obwohl die WaR. schwächer wurde, ja sogar für einige Zeit verschwand; 
der Zustand sich in keiner Weise besserte, mitunter sich sogar verschlimmerte, während wir leidliche Besserungen sahen, ohne daß die WaR. im Blut oder Liquor sich änderte. Nur in einem Falle sahen wir, da $\beta$ jedesmal, wenn Erregungszustände auftraten, die WaR. positiv wurde, während nach dem Abklingen der Erregungszustände die Wa.R. im Blut und Liquor negativ ausfiel.

Fall 27. Josef M. 36 Jahre.

Am 11. IIr. 1919 wurde er im schweren Frregungszustand aufgenommen, er wollte nach Koblenz, um mit den Amerikanern Frieden zu schließen, brachte zahlreiche Größenideen vor, Wir erfuhren, daß er 15 Jahre vor der Aufnahme eine Lues gehabt hat, daß er mehreren Kuren sich unterzogen hatte und 7 Jahre nach der Infektion mit Erlaubnis des Arztes heiratete. Aus der Ehe ist ein gesundes Kind, die Frau ist nicht infiziert. M. hat den ganzen Feldzug mitgemacht, ist bis zum Ende des Krieges nie aufgefallen. Vor der Aufnahme hat er noch eine energische Salvarsankur durehgemacht.

15. III. 1919. WaR. im Blut neg., im Liquor pos. 0,4.

14. V. 1919. WaR. im Blut neg., im Liquor neg.

17. VI. 1919. WaR. im Blut neg., im Liquor neg.

23. VII. 1919, WaR. im Blut neg., im Liquor neg.

8. IX. 1919. WaR. im Blut neg., im Liquor neg.

22. I. 1920. WaR. im Blut neg., im Liquor neg.

8. III. 1920. WaR. im Blut neg., im Liquor pos. 1,0.

23. IV. 1920. WaR. im Blat neg., im Liquor pos. 0,6.

15. V. 1920. WaR. im Blut neg., im Liquor pos. 0,4 .

20. VI. 1920. WaR. im Blut neg., im Liquor neg.

14. VIII. 1920. WaR. im Blut Andeutung von pos. Reaktion, im Liquor neg. 8. XI. 1920. WaR. im Blut neg., im Liquor neg.

10. I. 1920. WaR. im Blut Andeutung von pos. Reaktion, im Liquor pos. 0,6. 15. II. 1920. WaR. im Blut neg., im Liquor pos. 0,6.

Nun lag die Frage nahe, ob derartig schwankende Befunde durch die Treponemenbefunde zu klären sind, ob überhaupt zwischen den Treponemenbefunden und der Stärke der WaR. Beziehungen zu finden sind. F. Pulido Valente hat diese Frage schon eingehend an 40 Paralytikern studiert, er konnte bei 28 von 40 Fällen durch Hirnpunktion Treponemen nachweisen, und er stellte fest, daß in der Mehrzahl der Tälle der Liquor schwächer als das Serum, mitunter gleichstark, niemals aber stärker reagierte. In 5 Fällen reagierte der Liquor trotz Treponemenbefundes negativ, in 4 Fällen sehr schwach positiv; in allen 9 Fällen war die Blutreaktion positiv, in 8 Fällen sogar stark positiv.

Wir haben zur Beantwortung dieser Frage nur die Sektionsbefunde herangezogen, sofern wir kurz vor dem Tode Blut und Liquor auf WaR. untersucht hatten, und zwar sind vor allem die Fälle von Interesse, bei denen Treponemen im Hirn nachzuweisen waren. In einigen Fällen war wohl bei positivem Treponemenbefund eine sehr starke Blut- und Liquorreaktion zu finden, in anderen Fällen aber, wie z. B. Fall 14 u. ló zeigen, war die Blutreaktion nur 
sehr schwach positiv oder negativ und die Liquorreaktion schwach positiv, in einigen wenigen Fällen fanden wir aber trotz positiven Treponemenbefundes negative Blut- und Liquorreaktionen. Unsere Befunde decken sich also ungefähr mit den Befunden Valentes.

Wenn wir noch einmal alles kurz zusammenfassen, so müssen wir erklären, daß unsere Arbeit noch keine völlige Klärung aller gestellten Fragen bringt, daß vielmehr derartige Untersuchungen noch fortgesetzt werden müssen, und zwar erscheint es uns sehr wichtig, dieselben Fälle sehr häufig und über lange Zeiträume hin zu untersuchen und zwischen behandelten und unbehandelten Fällen zu unterscheiden.

Unsere Beobachtungen haben uns gezeigt, daß der Ausfall der WaR. bei manchen Paralytikern im Verlauf der Krankheit sehr schwanken kann. Auch wir konnten wie Kafka mehrere Fälle, bei denen von einer früheren Behandlung nichts bekannt war, beobachten, deren WaR. im Liquor stärker als im Blut war, ja sogar, wo bei starker Tiquorreaktion eine negative Blutreaktion gefunden wurde. Jedenfalls ergibt unser Material, daß die WaR. im Blut und Liquor der Paralytiker, auch der unbehandelten, nicht immer positiv ist, daß vielmehr die eine oder andere Reaktion fehlen kann.

Die Frage, ob die Reaktionen durch die verschiedensten Behandlungsarten beeinflußt werden, können wir dahin beantworten, daß durch jede Behandlungsart die WaR. im Blut und Liquor beeinflußt werden kann, daß aber die Blutreaktion leichter als die Liquorreaktion zu beeinflussen ist. Meist treten die Reaktionen nach kürzerer oder längerer Zeit zu ihrer alten Stärke zurück, doch konnten wir einige Fälle beobachten, wo die negative Blut- und Liquorreaktion seit Jahren besteht, es sind dies meist Fälle, die mit großen Salvarsandosen behandelt worden sind, oder bei denen durch die Fiebertherapie hohe Temperaturen erzielt worden waren.

Die Frage, ob aus dem Ausfall der Stärke der WaR. auf den Krankheitszustand zu schließen ist, müssen wir auf Grund unseres Materiales verneinen. Wir sahen Fälle, bei denen die Reaktionen schwächer oder negativ wurden, ohne daß der Zustand des Kranken sich änderte, wo sogar Verschlimmerungen zu erkennen waren, und wiederum Fälle, wo die Reaktionen in derselben Stärke blieben und die Kranken leidliche Remissionen bekamen. Nur in einem Fall sahen wir Beziehungen zwischen dem Ausfall der Reaktion und dem Krankheitszustande.

In der Frage, ob zwischen den Treponemenbefunden und der Stärke der WaR. Beziehungen bestehen, kommen wir zu denselben Ergebnissen wie Valente auf Grund seiner Himpunktionen. Trotz Treponemenbefunden können die Reaktionen schwach ausfallen oder sogar fehlen. 
318 E. Nathan u. R. Weichbrodt: Untersuch. über d. Wassermannsche Reakt. usw.

\section{Literatur,}

Blumenthal und Tsakalotis, Zur quantitativen Bestimmung der Syphilisstoffe. Dermatol. Zeitschr, 23. —- Jahne1, Über das Vorkommen von Spirochäten in der Aorta bei progr. Paralyse; Zeitschr. f. d. ges. Neurol. u. Psychiatr. Orig. 60. - Kafka, Atypische serologische Befunde bei Paralyse u. ihre Bedeutung. Zeitschr. f. d. ges. Neurol. u. Psychiatr. Orig. 56 u. 59. - Krüger, Ergebnisse der Blut- und Spinalflüssigkeituntersuchungen bei luetischen Hirn- $u$. Rückenmarkserkrankungen. Monatsschr. f. Psych. 48. - F. Lesser, Die praktische Bedeutung der quantitativen WaR. für die Behandlung des Syphilis. M. m. W. 1914. - Pla ut, Die WaR. der Paralyse. Zeitschr. f. d. ges. Neurol. u. Psychiatr. Orig. 56 u. 59. - F. Pulido Valente, Sur l'étiologie et la pathogénie de la Paralysie générale. Arguivos do Instituto Bacteriologico Camare Pistana Lissabòn 1918. - Weichbrodt, Therapie der Paralyse. Arch. f. Psych. 61. 